\section{Oculoplastic reconstruction following Mohs surgery}

\author{
Abstract
}

Purpose Mohs micrographic surgery has been advocated as the optimal management of nonmelanoma skin cancer in the periocular region. It is a technique that is ideally suited to the removal of skin tumours with a contiguous growth pattern such as basal cell carcinoma and squamous cell carcinoma, allowing examination of $100 \%$ of the surgical margin. As a result of this total margin control, the technique offers an unsurpassed cure rate combined with maximal preservation of normal tissue. Following excision of a periocular tumour by a Mohs surgeon, the resulting defect usually requires reconstruction. Our objective was to determine whether the size of defect produced by Mohs surgery and the type of reconstruction required differed from the results we would have expected from standard surgery with a $3 \mathrm{~mm}$ excision margin.

Methods A Mohs surgery service with a combined dermatological and oculoplastic approach was set up in Manchester in 1994. We reviewed 60 of our patients who underwent Mohs surgery and compared the size of defect produced as well as the type of reconstruction required with the results we would have predicted for standard excisional surgery with a $3 \mathrm{~mm}$ margin.

Results Although a minority of patients required larger reconstructions than would have been anticipated $(20 \%)$, many had smaller reconstructions than we had predicted (37\%). This latter group often had important
CLARE INKSTER, JANE ASHWORTH, JOHN R. MURDOCH, PETER MONTGOMERY, NICHOLAS R. TELFER, BRIAN LEATHERBARROW Squamous cell carcinoma, Eyelid reconstruction

Basal cell carcinoma is the most common periocular malignancy. Although rarely fatal, it may result in considerable morbidity. 'Tumours may cause severe cosmetic deformity and significantly compromise the effective function of the eyelids and lacrimal apparatus.

Squamous cell carcinoma is less common, but has a higher propensity for metastasis and a significant mortality rate. ${ }^{2,3}$ The search for the ideal treatment modality for non-melanoma skin cancer led Frederic Mohs to develop his technique of micrographic surgery in the 1930s and 1940s. ${ }^{4}$ The fresh tissue technique of micrographic surgery is well described elsewhere ${ }^{5-8}$ The unique feature of Mohs surgery is the use of horizontal rather than vertical tissue sections. This allows examination of $100 \%$ of the surgical margin, including the entire undersurface (deep margin) of the lesion and all the epithelial edges. Thin flat layers of tissue are excised in a stage-by-stage approach and immediately processed in a frozen section Mohs laboratory. The technique allows the location of any residual tumour to be accurately identified, thus sparing uninvolved tissue. By these means one can be assured, with a high degree of certainty, of the complete excision of the tumour without undue sacrifice of adjacent normal tissue and uninvolved structures such as the lacrimal canaliculi.

A Mohs surgery service was established in Manchester in 1994. The service involves a combined dermatological and oculoplastic approach to the management of periocular tumours, as has been previously advocated., 10 The patient's tumour is excised by a dermatologist trained in Mohs surgery. The resultant defect is then reconstructed by an oculoplastic surgeon for the best cosmetic and functional result.

We looked at the first 60 patients managed in this joint manner who had a complete photographic record. Our objective was to determine whether the size of defect produced and the type of reconstruction required differed from the results we would have expected from 
Table 1. Tumour location

\begin{tabular}{lc}
\hline Location of tumour & No. of patients \\
\hline Lower lid & $27(42 \%)$ \\
Upper lid & $10(16 \%)$ \\
Medial canthus & $21\left(33^{\prime} \%\right)$ \\
Lateral canthus & $6(9 \%)$ \\
More than one location & $+(7 \%)$ \\
\hline
\end{tabular}

standard excisional surgery. We defined standard surgery in the periocular region as the excision of a $3 \mathrm{~mm}$ margin of healthy tissue around the clinical margin of the tumour. Where the tumour involved the eyelid margin, we assumed that a full-thickness excision would have been necessary without Mohs surgery.

\section{Patients and methods}

Since the beginning of 1994, all patients with periocular basal cell and squamous cell carcinomas referred to either the dermatology (N.R.T.) or oculoplastic (B.L.) consultants in Manchester have been offered a combined Mohs surgery excision and oculoplastic reconstruction of their tumours. They are provided with an information leaflet explaining the aims of the joint approach. Patients who choose this approach are seen by both teams preoperatively. The excision of the tumour is carried out in the dermatology department as a day-case procedure, under local anaesthesia. The patient is assessed later the same day by the oculoplastic team to determine the type of reconstruction required. Depending on the patients' social circumstances, they are then either allowed home or admitted to the Eye Hospital. The reconstruction is performed the following day under local or general anaesthesia depending on the type of reconstruction required. The patient is followed up by the oculoplastic team until all wounds have settled, and is kept under long-term review by the dermatologists. Clinical photographs are taken of the tumour, the defect following Mohs excision, and the final post-operative result.

In this study, we reviewed the first 60 patients managed in this joint manner who had a complete photographic record. As we were unable to locate a complete photographic record on 7 of the first 60 patients, we included the next 7 patients chronologically with such a record. The photographic appearance of the tumour for each patient was then shown to an experienced oculoplastic fellow (P.M.) who had not been involved in the care of these patients. He made an attempt to delineate the clinical margin of the tumour, and estimated the area and depth of tissue that would need to be excised to produce a $3 \mathrm{~mm}$ excision margin. He then documented the type of reconstruction that would be needed to repair his predicted defect. He also recorded whether he would have needed to sacrifice any important structures in the periocular region. We defined important structures as lacrimal canaliculi, canthal tendons or posterior lamellae of the eyelids. The oculoplastic fellow was then shown a photograph of the actual tissue defect produced by Mohs surgical excision. He was asked to comment if this was greater, less than, or the same as his predicted defect, in terms of both area of skin loss and depth of tissue taken. As this was inevitably an estimate, we recorded it as greater or less than predicted only if he felt the difference was clinically significant. For example, in the case of a small medial canthal tumour, he did not predict that it would be necessary to excise tissue down to periosteum to achieve a tumour-free margin. We then compared his predicted sacrifice of important structures, and predicted type of reconstruction, with the surgical notes. We also looked at other parameters including the age and sex distribution of the patients, tumour histology, whether tumours were primary or recurrent, the amount of theatre time used, and the type of anaesthesia required for the reconstruction.

\section{Results}

Of the 60 patients reviewed, $29(48 \%)$ were male and 31 $(52 \%)$ were female. Their ages ranged from 34 to 91 years with a mean age of 68 years.

Twelve patients $(20 \%)$ had recurrent tumours. The original tumour in these cases had been treated by a variety of means including simple excision, cryotherapy and radiotherapy. The time taken from initial treatment to documentation of recurrence ranged from 2 weeks to 11 years. Three tumours $(5 \%)$ were squamous cell carcinomas and the rest were basal cell carcinomas.

Reconstructions were performed under local anaesthesia in 50 cases $(83 \%)$ and under general anaesthesia in 10 cases $(17 \%)$. Twenty-nine patients $(48 \%)$ were treated on a day-case basis and $31(52 \%)$ required an overnight admission. The amount of theatre time used ranged from $19 \mathrm{~min}$ to $185 \mathrm{~min}$ with a mean of $83 \mathrm{~min}$. This does not include any time required for second-stage procedures such as flap division.

The tumours were initially classified according to their location (Table 1). The photographs were then examined as discussed above. There did not appear to be any discernible trend relating size of defect to location. The results of the comparison between actual and predicted defects are shown in Table 2. Important structures such as the canaliculi, canthal tendons or posterior lamellae were preserved contrary to prediction in 15 cases $(25 \%)$, and lost in only $2(3 \%)$. Fig. 1 shows a

Table 2. Comparison of patients' actual and predicted outcome's

\begin{tabular}{lccc}
\hline & Greater than anticipated & Less than anticipated & Same as anticipated \\
\hline Area of skin loss & $23(38 \%)$ & $15(25 \%)$ & $22(37 \%)$ \\
Depth of tissue taken & $11(18 \%)$ & $15(25 \%)$ & $34(57 \%)$ \\
Size of reconstruction & $12(20 \%)$ & $22(37 \%)$ & $26(43 \%)$ \\
\hline
\end{tabular}




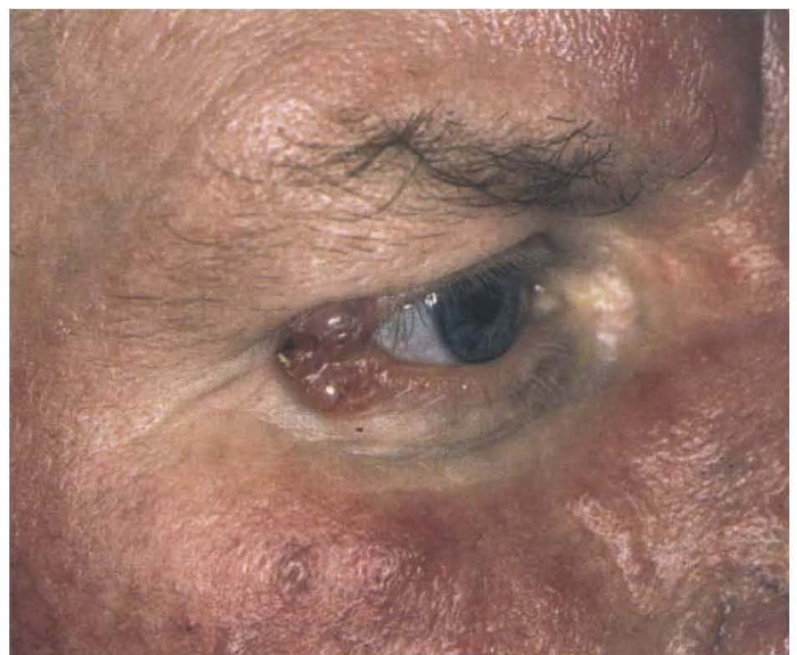

(a)

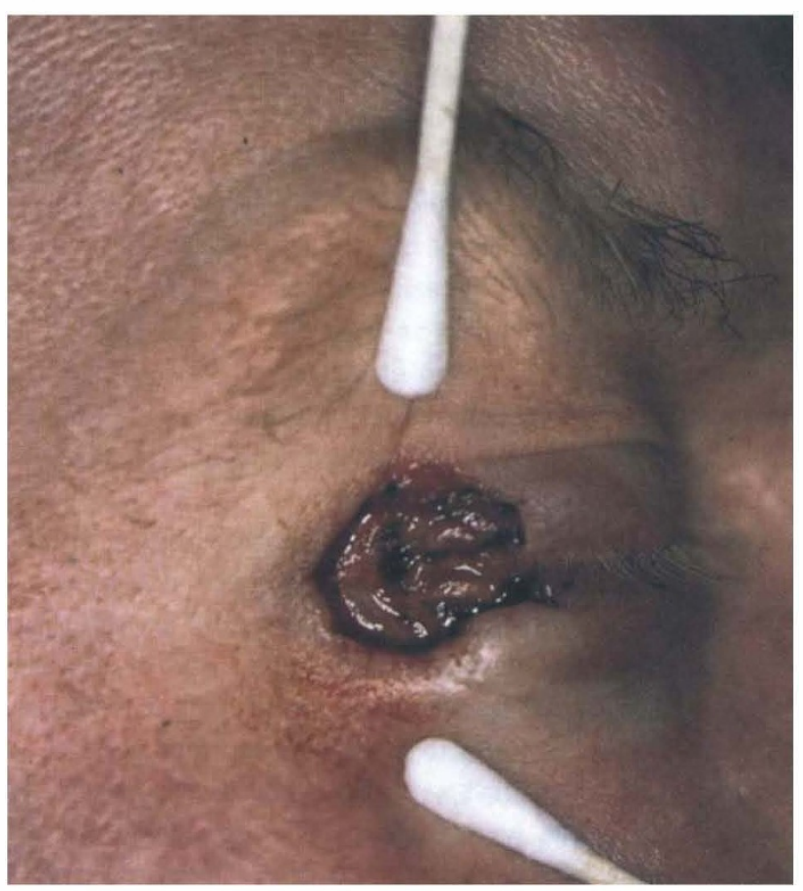

(b)

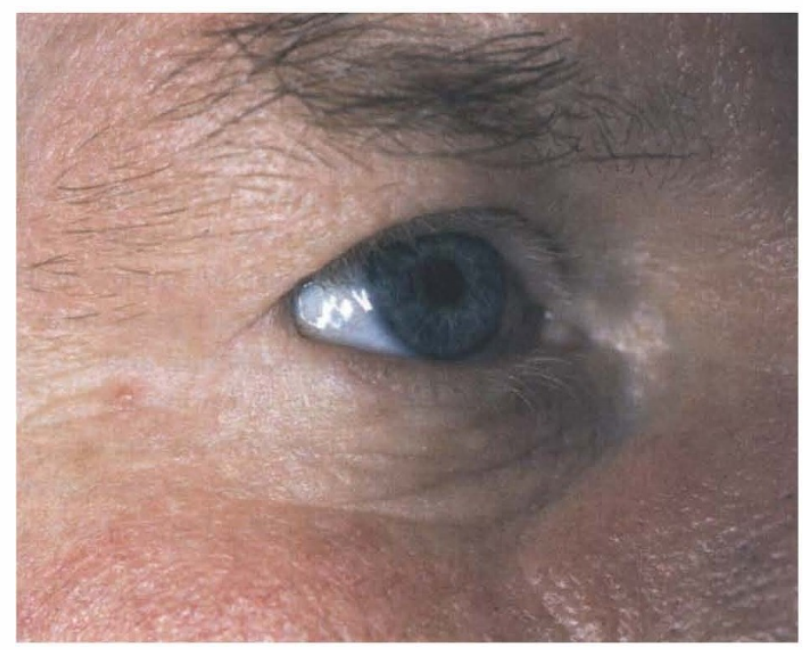

(c)

Fig. 1. (a) Large basal cell carcinoma at the right lateral canthus. (b) Defect following Mohs excision - smaller than anticipated. 67-year-old man with a large basal cell carcinoma at the right lateral canthus. Here it had been predicted that the lateral canthal tendon and part of the posterior lamella of the upper lid would have had to be excised with standard surgery. This would have required periosteal flaps to recreate the lateral canthal tendon and posterior lamella, followed by an upper lid advancement flap and lower lid cheek flap. Mohs surgery did not detect any subclinical tumour extension and therefore a simple advancement flap sufficed. In another case, a 32-year-old man presented with a basal cell carcinoma at his right medial canthus. It was predicted that standard excisional surgery with a $3 \mathrm{~mm}$ margin would have required removal of both upper and lower lacrimal canaliculi. In fact Mohs surgery allowed these to be preserved.

It was assumed that patients who had more tissue taken than predicted would have had tumour cells missed with standard excisional surgery, and would therefore have had the potential for recurrence. Fig. 2 demonstrates one such case. In this study 24 patients ( $40 \%$ ) had either a greater area of skin or depth of tissue taken, and therefore would have had a potential for recurrence with standard excision. Of the 12 patients who had recurrent tumours at presentation, $8(67 \%)$ had larger reconstructions that predicted. An example involves the case of a 53-year-old woman who developed a clinically small area of tumour recurrence at the lower border of her right lower lid, having had previous excision of a basal cell carcinoma. At Mohs surgery she was found to have a large area of subclinical tumour extension, which involved much of the skin of her lower lid and upper cheek. It had been predicted that she would require a small full-thickness skin graft if a $3 \mathrm{~mm}$ excision margin had been allowed. In fact she required a large cheek rotation flap, with a split-thickness skin graft to the pre-auricular area.

There were no complications arising from the surgical reconstruction, but there was one interesting problem that occurred during the initial Mohs excision. This involved a woman who had previously undiagnosed basal cell naevus syndrome. In her case tumour-free margins could not be obtained as nests of tumour cells were present extensively across her face.

\section{Discussion}

Mohs micrographic surgery has provided a means of removing periocular basal cell and squamous cell carcinoma with an unsurpassed cure rate while producing the minimum of unnecessary tissue deficit. ${ }^{11}$ Previous studies have documented cure rates as high as $99 \%$ for primary tumours, ${ }^{12}$ and $94 \%$ for recurrent tumours. ${ }^{13}$ As a time-consuming procedure Mohs surgery must be justified by its cure rate. As a result less emphasis has been placed on the tissue conservation aspects of the treatment. It has been noted before, however, that reconstructions following periocular Mohs surgery are frequently smaller than would be expected with standard surgical excision. ${ }^{14}$ This concurs with the results of our study, which showed that $37 \%$ of patients 


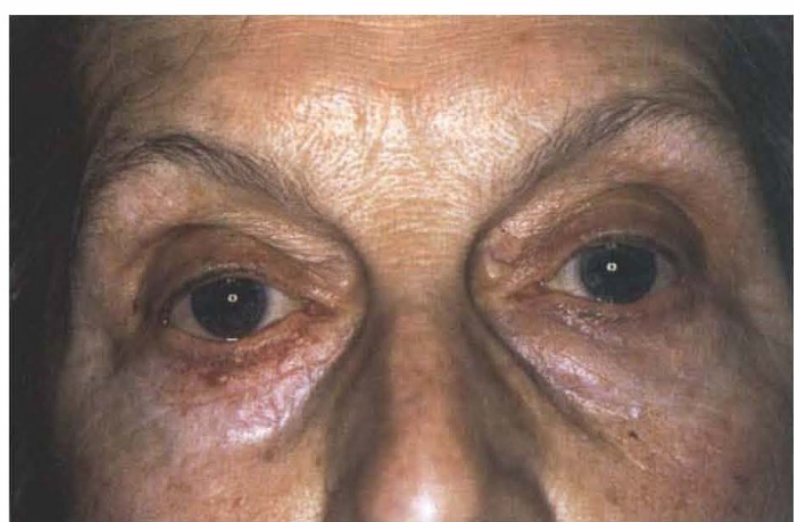

(a)

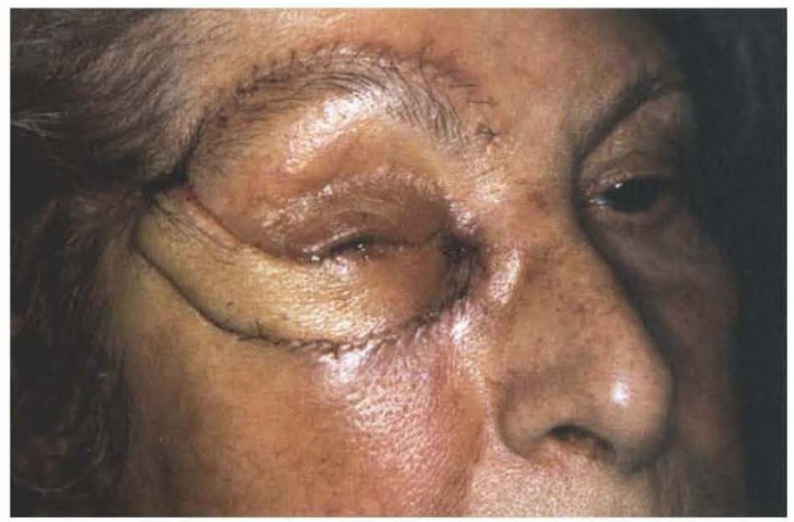

(c)

Fig. 2. (a) A morphoeic right lower lid basal cell carcinoma. (b) Defect following Mohs excision-larger than anticipated. (c) Appearance following first stage of Fricke flap reconstruction. (d) Final post-operatio' apperarance.

required smaller reconstructions than anticipated. It does not seem unreasonable to propose that the $20 \%$ of patients who had larger reconstructions with Mohs surgery still benefited in the long term. These are the patients who would have had tumour cells missed by standard surgical excision with a $3 \mathrm{~mm}$ margin. They would therefore have stood a high chance of requiring further surgery to deal with recurrent tumour. In our series $20 \%$ of patients had in fact already experienced unsuccessful treatment of their tumour by other means. Overall 38 of the 60 patients $(64 \%)$ appeared to benefit from treatment with Mohs surgery as they had either smaller reconstructions than they would have had, or a lower potential for recurrence. Seven patients in this group benefited doubly in that they required a larger area of skin to be excised than anticipated but a shallower depth of tissue was taken and therefore a much simpler reconstruction sufficed.

In this study there was an inevitable degree of subjectivity in the clinical estimate of the defect that would have been produced with a standard surgical excision including a $3 \mathrm{~mm}$ margin. In order to offset such bias, the photographs were assessed by an experienced oculoplastic fellow (P.M.) who had not been present at the time these patients were treated. It should also be commented that making clinical decisions from photographs can be misleading.

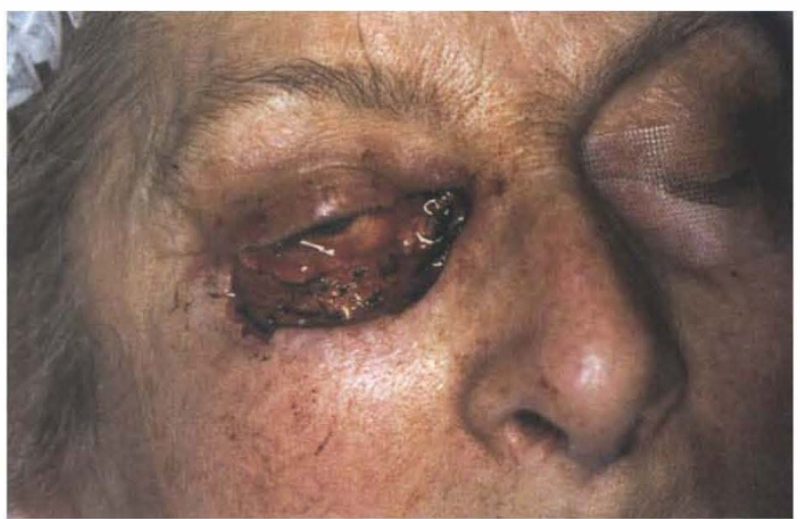

(b)

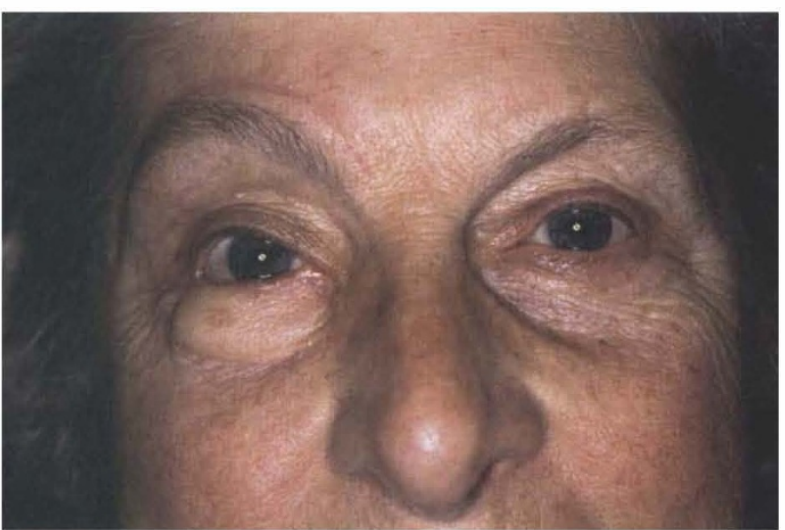

(d)
There are several clinical implications of our results Patients who require smaller reconstructions can often benefit from day-case care or a shorter inpatient stay. There is less use of theatre time, and fewer two-stage procedures are likely to be required. Fewer general anaesthetics are required and fewer follow-up visits. Better cosmetic and functional results are generally obtained for the patients. As a consequence, the costs involved in the reconstructive surgery are reduced. In addition the cost of dealing with recurrent tumours should be much reduced. Patients who have larger reconstructions may need general anaesthesia, so fitness for this should be assessed prior to excision of the lesion, even if it appears small clinically. In addition, greater surgical expertise may be required, so the Mohs surgeon should have good communication with his oculoplastic colleague.

In conclusion, Mohs micrographic surgery offers the dual advantages of maximal cure rate and maximal preservation of normal tissue, and as such is the optimal surgical approach for the removal of periocular basal cell and squamous cell carcinoma. Best results are obtained with a combined dermatological and oculoplastic approach. At present the availability of this treatment modality in the United Kingdom is limited by a lack of appropriately trained Mohs surgeons. The oculoplastic expertise required already exists in many centres in this country. Referral for Mohs surgery should be considered 
for all large or recurrent lesions, morphoeic lesions (in which the clinical margins of the tumour are difficult to define), medial canthal lesions and all lesions in younger patients.

\section{References}

1. Randle HW. Basal cell carcinoma: identification and treatment of the high-risk patient. Dermatol Surg 1996;22:255-61.

2. Tavin E, Persky M. Metastatic cutaneous squamous cell carcinoma of the head and neck region. Laryngoscope 1996;106:156-8.

3. Rowe DE, Carroll RJ, Day CL Jr. P'rognostic factors for local recurrence, metastasis, and survival rates in squamous cell carcinoma of the skin, ear, and lip: implications for treatment modality selection. J Am Acad Dermatol 1992;26:976-90.

4. Mohs FE. Chemosurgery: a microscopically controlled method of cancer excision. Arch Surg 1941;42:279-95.

5. Mohs FE. Cancer of the eyelids. Bull Am Coll Chemosurg 1970;3:10-1.

6. Tromovitch TA, Stegman SJ. Microscopically controlled excision of skin tumours: chemosurgery (Mohs); fresh tissue technique. Arch Dermatol 1974;110:231-2.
7. Tromovitch TA, Stegman SJ. Microscope-controlled excision of cutaneous tumours: chemosurgery, fresh tissue technique. Cancer 1978;41:653-8.

8. Dzubow LM. Mohs surgery. Lancet 1994;343:433-4.

9. Anderson RL, Ceilly RI. A multi-specialty approach to the excision and reconstruction of eyelid tumours. Ophthalmology 1978;85:1150-63.

10. Irvine C, Walker NP, Ramnarain NI), Downes RN, Collin JR. Micrographically controlled excision (Mohs surgery) of basal cell carcinoma around the eye. Combined dermatological surgical clearance and oculoplastic surgical repair. Aust N Z J Ophthalmol 1992;20:5-10.

11. Leshin B, Yeatts P, Anscher M, et al. Management of periocular basal cell carcinoma: Mohs micrographic surgery versus radiotherapy. Surv Ophthalmol 1993;38:193-212.

12. Rowe DE, Carroll RJ, Day CL Jr. Long-term recurrence rates in previously untreated primary basal cell carcinoma: implications for patient follow-up. J Dermatol Surg Oncol 1989;15:315-28.

13. Rowe DE, Carroll RJ, Day CL Jr. Mohs surgery is the treatment of choice for recurrent (previously treated) basal cell carcinoma. J Dermatol Surg Oncol 1989;15:424-31.

14. Downes RN, Walker NP, Collin JR. Micrographic (Mohs) surgery in the management of periocular basal cell epitheliomas. Eye 1990;4:160-8. 